\title{
The Metric Operator and the Functional Integral Formulation of Pseudo-Hermitian Quantum Mechanics
}

\author{
H. F. Jones
}

Pseudo-Hermitian quantum theories are those in which the Hamiltonian $H$ satisfies $H^{\dagger}=\eta \eta^{-1}$, where $\eta \equiv e^{-Q}$ is a positive-definite Hermitian operator, rather than the usual $H^{\dagger}=H$. In the operator formulation of such theories the standard Hilbert-space metric must be modified by the inclusion of $\eta$ in order to ensure their probabilistic interpretation. With possible generalizations to quantum field theory in mind, it is important to ask how the functional integral formalism for pseudo-Hermitian theories differs from that of standard theories. It turns out that here Q plays quite a different role, serving primarily to implement a canonical transformation of the variables. It does not appear explicitly in the expression for the vacuum generating functional. Instead, the relation to the Hermitian theory is encoded via the dependence of $Z$ on the external source $j(t)$. These points are illustrated and amplified in various versions of the Swanson model, a non-Hermitian transform of the simple harmonic oscillator.

Keywords: quantum mechanics, functional integral, non-Hermitian.

\section{Introduction}

\subsection{The metric operator in the Schrödinger formulation of pseudo-Hermitian quantum mechanics}

The recent interest in non-Hermitian Hamiltonians stems from the work of Bender and Boettcher [1], who showed numerically that the class of Hamiltonians

$$
H=\frac{1}{2} p^{2}-g(i x)^{N}
$$

has a completely real spectrum for $N \geq 2$. They attributed this property to an unbroken $\mathcal{P} \mathcal{T}$-symmetry, whereby

$$
x \rightarrow-x, \quad i \rightarrow-i \text {. }
$$

A rigorous proof [2] of the reality came a few years later by exploiting the ODE-IM correspondence, i.e. the correspondence between ordinary differential equations in their different Stokes sectors and integrable models.

In such cases there exists a similarity transformation from the non-Hermitian $H$ to a Hermitian $h$ :

$$
h=\rho H \rho^{-1} \text {. }
$$

Here $\rho$ is a positive-definite Hermitian operator (re)introduced by Mostafazadeh [3].

It is related to the $Q$ operator [4], which provides a positive-definite metric for the quantum mechanics governed by $H$, according to

$$
\rho=e^{-\frac{1}{2} Q} .
$$

It is also useful to introduce its square

$$
\eta \equiv \rho^{2}=e^{-Q}
$$

From Eq. (3)

$$
\rho^{-1} H^{\dagger} \rho=h^{\dagger}=h=\rho H \rho^{-1} .
$$

So

$$
H^{\dagger}=\rho^{2} H \rho^{-2}=\eta H \eta^{-1} .
$$

This replaces the usual Hermiticity requirement on the Hamiltonian. $H$ is said to be quasi-Hermitian [5], or pseudo-Hermitian ${ }^{(1)}$, with respect to $\eta$.

The operator $\eta \equiv e^{-Q}$ is in fact precisely the metric operator occurring in

$$
\langle\langle\psi, A \varphi\rangle\rangle \equiv\langle\psi, \eta A \varphi\rangle,
$$

because the similarity transformation $A^{\prime}=\rho A \rho^{-1},\left|\psi^{\prime}\right\rangle=\rho|\psi\rangle$ gives

$$
\left\langle\psi^{\prime}\left|A^{\prime}\right| \varphi^{\prime}\right\rangle=\left\langle\psi\left|\rho^{\dagger} \rho A \rho^{-1} \rho\right| \varphi\right\rangle .
$$

Here $\rho^{\dagger} \rho=\rho^{2}=\eta$, rather than 1 , as would be the case if $\rho$ were unitary rather than Hermitian, so

$$
\left\langle\psi^{\prime}\left|A^{\prime}\right| \varphi^{\prime}\right\rangle=\langle\psi|\eta A| \varphi\rangle \text {. }
$$

If the operator $A^{\prime}$ is Hermitian then $A$ is pseudo-Hermitian: $A^{\dagger}=\eta A \eta^{-1}$, and is an observable, with real eigenvalues (the same as those of $A^{\prime}$ ).

\subsection{Functional integral formalism of quantum mechanics}

In the functional integral formulation of standard Hermitian quantum mechanics, the basic object of interest is the vacuum generating functional

$Z[j]=\int[D \varphi] \exp \left\{-\int \mathrm{d} t\left[\frac{1}{2} \dot{\varphi}^{2}+V(\varphi)-j(t) \varphi\right]\right\}$,

from which Green functions can be obtained by functional differentiation with respect to $j(t)$.

The fundamental question we are asking here is, what is the corresponding expression in pseudo-Hermitian quantum mechanics? One might perhaps expect something like

$Z[j]=\int[D \phi] \eta \exp \left\{-\int \mathrm{d} t\left[\frac{1}{2} \dot{\varphi}^{2}+V(\varphi)-j(t) \varphi\right]\right\}$,

but this is not what happens. In fact $\eta$ does not appear explicitly in the expression for $Z[j]$. Rather, depending on the 
metric chosen in the operator formalism, $j$ will appear differently in the Lagrangian.

The method we will use to find the correct expression for $Z$ is to use the similarity transformation between our pseudo-Hermitian theory and the equivalent Hermitian theory, where we know how $Z$ should be written. In this paper we will limit ourselves to a particular soluble model, the Swanson model, which can be formulated as a viable quantum theory in a variety of ways (in fact there is a one-parameter family [6] of $\eta \mathrm{s})$, of which we will pick the three simplest. In [7] we treated the two cases $Q=Q(x)$ and $Q=Q(p)$, and in addition the pseudo-Hermitian "wrong-sign" quartic oscillator, i.e. Eq. (1) for $N=4$.

\section{$2 Z$ for various versions of the Swanson model}

The Hamiltonian for this model, first introduced in [8], is

$$
H=\omega a^{\dagger} a+\alpha a^{2}+\beta a^{\dagger^{2}}
$$

where $a$ and $a^{\dagger}$ are standard lowering and raising operators for a simple harmonic oscillator with unit frequency, and $\omega, \alpha$ and $\beta$ are real parameters. $H$ is non-Hermitian for $\alpha \neq \beta$. In terms of $x$ and $p$,

$$
H=a x^{2}+b p^{2}+c\{x, p\}_{+},
$$

where $a=\frac{1}{2}(\omega+\alpha+\beta), b=\frac{1}{2}(\omega-\alpha-\beta), c=\frac{1}{2} i(\alpha-\beta)$.

\section{$2.1 Q=Q(x)$}

$H$ can be written as

$H(x, p)=\left(a-\frac{c^{2}}{b}\right) x^{2}+b\left(p+\frac{c}{b} x\right)^{2} \equiv \widetilde{a} X^{2}+b P^{2}=h(X, P)$

This is a simple harmonic oscillator with frequency $\Omega=2 \sqrt{\widetilde{a} b}$.

Recall that $H(x, p)=e^{\frac{1}{2} Q} h(x, p) e^{-\frac{1}{2} Q}$. So $Q$ has to satisfy

$$
\begin{aligned}
X & \equiv x=e^{\frac{1}{2} Q} x e^{-\frac{1}{2} Q}, \\
P & \equiv p+\frac{c}{b} x=e^{\frac{1}{2} Q} p e^{-\frac{1}{2} Q},
\end{aligned}
$$

which can be achieved by

$$
Q=-\frac{i c}{b} x^{2} .
$$

Note that Eq. (13) represents a (complex) canonical transformation between the pairs $(x, p)$ and $(X, P)$. Classically $Q$ appears as the active part of the generator

$$
F_{2}(x, P) \equiv x P-\frac{1}{2} i Q(x)
$$

of this canonical transformation, according to which

$$
X=\frac{\partial F_{2}}{\partial P}=x, \quad p=\frac{\partial F_{2}}{\partial x}=P-\frac{1}{2} i Q^{\prime} .
$$

It is also worth noting that to construct the classical Lagrangian corresponding to Eq. (11) we have

$$
L \equiv \dot{x} p-H=\frac{\dot{x}^{2}}{4 b}-\widetilde{a} x^{2}-\frac{c}{b} x \dot{x}
$$

which differs from a normal (scaled) Lagrangian for the harmonic oscillator with frequency $\Omega$ only by the total derivative $(c / b) x \dot{x}=\frac{1}{2} i \dot{Q}$.

Our approach will be to start with the naive form for the Euclidean $Z[0]$ corresponding to $H$, verify that this is correct by transforming to its Hermitian equivalent, then insert the external source $j(t)$ coupled to the Hermitian observable, and finally transform back to obtain the form of $Z[j]$ for the non-Hermitian Hamiltonian $H$. In this spirit we suppose that $Z[0]=\int[D \varphi][D \pi] \exp \left\{-\int \mathrm{d} t\left[i \dot{\varphi} \pi+a \varphi^{2}+b \pi^{2}+2 c \varphi \pi\right]\right\}$,

in which we have $H$ written in terms of $\varphi$ and $\pi$.

We then complete the square in exactly the same way as in Eq. (12), to obtain

$Z[0]=\int[D \varphi][D \Pi] \exp \left\{-\int \mathrm{d} t\left[i \dot{\varphi}\left(\Pi-\frac{c}{b} \varphi\right)+\widetilde{a} \varphi^{2}+b \Pi^{2}\right]\right\}$,

with $\Pi=\pi+\frac{c}{b} \varphi$. Here the term $-i \frac{c}{b} \dot{\varphi} \varphi$ in the exponent is precisely $\frac{1}{2} \dot{Q}$, which can be neglected under the $t$ integration.

This is the only place that $Q$ makes its appearance in this procedure.

$$
\begin{aligned}
Z[0] & =\int[D \varphi][D \Pi] \exp \left\{-\int \mathrm{d} t\left[-i \ddot{\varphi} \Pi+\widetilde{a} \varphi^{2}+b \Pi^{2}\right]\right\} \\
& =\int[D \varphi] \exp \left\{-\int \mathrm{d} t\left[\frac{\dot{\varphi}^{2}}{4 b}+\widetilde{a} \varphi^{2}\right]\right\},
\end{aligned}
$$

(to be compared with the non-Euclidean Eq. (16)).

Now let us couple $j$ to the observable $\Phi \equiv \varphi$ in Eq. (19) and work backwards, to obtain

$$
\begin{aligned}
& Z[j]=\int[D \varphi] \exp \left\{-\int \mathrm{d} t\left[\frac{\dot{\varphi}^{2}}{4 b}+\widetilde{a} \varphi^{2}-j \varphi\right]\right\} \\
& =\int[D \varphi][D \pi] \exp \left\{-\int \mathrm{d} t\left[i \dot{\varphi} \pi+a \varphi^{2}+b \pi^{2}+2 c \varphi \pi-j \varphi\right]\right\} .
\end{aligned}
$$

Thus functional derivatives $\partial / \partial j$ bring down factors of the observable $\varphi$, and $Q$ does not appear at all!

\section{$2.2 Q=Q(p)$}

$H$ can equally be written as

$$
\begin{aligned}
H(x, p) & =a\left(x+\frac{c}{a} p\right)^{2}+\left(b-\frac{c^{2}}{a}\right) p^{2} \\
& \equiv a X^{2}+\widetilde{b} P^{2}=h(X, P) .
\end{aligned}
$$

This is again a simple harmonic oscillator with the same frequency $\Omega$, since $a \widetilde{b}=\widetilde{a} b$. In this case $Q$ has to satisfy

$$
\begin{aligned}
P & \equiv p=e^{\frac{1}{2} Q} p e^{-\frac{1}{2} Q} \\
X & \equiv x+\frac{c}{a} p=e^{\frac{1}{2} Q} x e^{-\frac{1}{2} Q},
\end{aligned}
$$

which can be achieved by 


$$
Q=\frac{i c}{a} p^{2}
$$

The corresponding classical generating function is

giving

$$
F_{2}(x, P) \equiv x P+\frac{1}{2} i Q(P)
$$

$$
p=\frac{\partial F_{2}}{\partial x}=P \quad X=\frac{\partial F_{2}}{\partial P}=x+\frac{1}{2} i Q^{\prime} .
$$

We now mimic this procedure in the functional integral, starting again with

$$
Z[0]=\int[D \varphi][D \pi] \exp \left\{-\int \mathrm{d} t\left[i \dot{\varphi} \pi+a \varphi^{2}+b \pi^{2}+2 c \varphi \pi\right]\right\},
$$

and completing the square in the manner of Eq. (21) rather than (12). Then

$Z[0]=\int[D \Phi][D \pi] \exp \left\{-\int \mathrm{d} t\left[i \pi\left(\dot{\Phi}-\frac{c}{a} \dot{\pi}\right)+a \Phi^{2}+\widetilde{b} \pi^{2}\right]\right\}$,

where $\Phi=\varphi+\frac{c}{a} \pi$. Again the term $i \frac{c}{a} \pi \dot{\pi}$ in the exponent is just $\frac{1}{2} \dot{Q}$, and can be dropped under the $t$ integration. So

$$
\begin{aligned}
Z[0] & =\int[D \Phi][D \pi] \exp \left\{-\int \mathrm{d} t\left[i \dot{\Phi} \pi+a \Phi^{2}+\widetilde{b} \pi^{2}\right]\right\} \\
& =\int[D \Phi] \exp \left\{-\int \mathrm{d} t\left[\frac{\dot{\Phi}^{2}}{4 \widetilde{b}}+a \Phi^{2}\right]\right\} .
\end{aligned}
$$

Now we restore $j$, coupled to the observable $\Phi$ in this Hermitian version and work backwards:

$Z[j]=\int[D \Phi][D \pi] \exp \left\{-\int \mathrm{d} t\left[i \dot{\Phi} \pi+a \Phi^{2}+\widetilde{b} \pi^{2}-j \Phi\right]\right\}$.

Rewriting this in terms of the original field $\varphi=\Phi-\frac{c}{a} \pi$, the square bracket in the exponent becomes (up to total derivatives)

$$
\begin{aligned}
{[]=} & i \ddot{\varphi} \pi+b \pi^{2}+a \varphi^{2}+2 c \varphi \pi-j \varphi-j \frac{c}{a} \pi \\
= & b\left[\pi+\frac{1}{2 b}\left(i \ddot{\varphi}+2 c \varphi-\frac{c}{a} j\right)\right]^{2}+\frac{1}{4 b} \dot{\varphi}^{2} \\
& +a \varphi^{2}-\frac{\Omega^{2}}{4 a b} j \varphi+\frac{i c}{2 a b} j \ddot{\varphi}-\frac{c^{2}}{4 a^{2} b} j^{2} .
\end{aligned}
$$

Integrating over $\pi$ and rescaling $\varphi \rightarrow \varphi \sqrt{2 b}$, the final result corresponding to the operator theory with metric given by $Q(p)$ is (note that had we coupled $j$ to $\varphi$ in (28), we would have simply reproduced (20))

$Z[j]=\int[D \varphi] \exp \left\{-\int \mathrm{d} t\left[\begin{array}{l}\frac{1}{2} \dot{\varphi}^{2}+\frac{1}{2} \Omega^{2} \varphi^{2}-\frac{\Omega^{2}}{2 a} \frac{j}{\sqrt{2 b}} \varphi \\ +\frac{i c}{a} \frac{j}{\sqrt{2 b}} \dot{\varphi}-\frac{c^{2} j^{2}}{4 a^{2} b}\end{array}\right]\right\}$
Again $Q$ does not appear explicitly, but now the source $j$ appears in an unfamiliar way, with terms in $j \ddot{\varphi}$ and $j^{2}$.

As a check of these results let us calculate the expectation values $\langle\Phi\rangle$ and $\left\langle\Phi_{1} \Phi_{2}\right\rangle$ from the expression (30).

The first is rather trivial:

$$
\langle\Phi\rangle=\left.\frac{1}{Z} \frac{\delta Z}{\delta j}\right|_{j=0}=\frac{1}{\sqrt{2 b}}\left\langle\frac{\Omega^{2}}{2 a} \varphi-\frac{i c}{a} \dot{\varphi}\right\rangle=0,
$$

as expected. However, the second check is more interesting:

$$
\begin{aligned}
\left\langle\Phi_{1} \Phi_{2}\right\rangle= & \left.\frac{1}{Z} \frac{\delta^{2} Z}{\delta j_{1} \delta j_{2}}\right|_{j=0} \\
= & \frac{1}{2 b}\left\langle\left(\frac{\Omega^{2}}{2 a} \varphi_{1}-\frac{i c}{a} \dot{\varphi}_{1}\right)\left(\frac{\Omega^{2}}{2 a} \varphi_{2}-\frac{i c}{a} \dot{\varphi}_{2}\right)\right\rangle \\
& +\frac{c^{2}}{2 a^{2} b} \delta\left(t_{1}-t_{2}\right) .
\end{aligned}
$$

But

$$
\begin{aligned}
\left\langle\varphi_{1} \varphi_{2}\right\rangle & =\frac{1}{2 \Omega} e^{-\Omega\left|t_{1}-t_{2}\right|} \\
\left\langle\dot{\varphi}_{1} \varphi_{2}\right\rangle & =-\left\langle\varphi_{1} \dot{\varphi}_{2}\right\rangle=\frac{1}{2} \varepsilon\left(t_{2}-t_{1}\right) e^{-\Omega\left|t_{1}-t_{2}\right|} \\
\left\langle\dot{\varphi}_{1} \dot{\varphi}_{2}\right\rangle & =-\frac{\Omega}{2} e^{-\Omega\left|t_{1}-t_{2}\right|}+\delta\left(t_{1}-t_{2}\right),
\end{aligned}
$$

giving

$$
\left\langle\Phi_{1} \Phi_{2}\right\rangle=\frac{\Omega}{4 a} e^{-\Omega\left|t_{1}-t_{2}\right|}=\frac{\widetilde{b}}{\Omega} e^{-\Omega\left|t_{1}-t_{2}\right|},
$$

which is indeed the result to be expected from Eq. (27).

$$
2.3 Q=Q\left(x^{2}+p^{2}\right)
$$

This was in fact the original similarity transformation found by Geyer et al. [9], according to which $Q=-\mu\left(x^{2}+p^{2}\right)$, with $\mu=\ln \frac{\alpha}{\beta}$. In this case the result of the similarity transfor-

$$
\begin{aligned}
\text { mation } e^{-\frac{1}{2} Q} & \ldots e^{\frac{1}{2} Q} \text { is } \\
X & =x \cosh \mu+i p \sinh \mu \\
P & =p \cosh \mu-i x \sinh \mu,
\end{aligned}
$$

resulting in $H(x, p)=h(X, P)=\kappa X^{2}+\lambda P^{2}$, where

$$
\begin{aligned}
& \kappa=\frac{1}{2}(\omega+2 \sqrt{\alpha \beta}), \\
& \lambda=\frac{1}{2}(\omega-2 \sqrt{\alpha \beta}) .
\end{aligned}
$$


In this case, the total derivative dropped under $\int \mathrm{d} t$ was not a multiple of $\dot{Q}$. It turns out that this was a special case when $Q$ was a quadratic in either $x$ or $p$. The more general result is that the two Lagrangians differ by the time derivative of

$$
\frac{1}{2}\left(\frac{x \partial Q(x, P)}{\partial x}-\frac{P \partial Q(x, P)}{\partial P}\right) .
$$

We have again checked that we correctly obtain $\langle\Phi\rangle$ and $\left\langle\Phi_{1} \Phi_{2}\right\rangle$ from functional derivatives of $Z[j]$.

\section{Discussion}

The essential formula is

$$
\begin{aligned}
Z[0] & =\int[D \varphi][D \pi] \exp \left\{-\int \mathrm{d} t[i \ddot{\varphi} \pi+H(\varphi, \pi)]\right\} \\
& =\int[D \Phi][D \Pi] \exp \left\{-\int \mathrm{d} t[i \ddot{\varphi} \pi+h(\Phi, \Pi)]\right\} .
\end{aligned}
$$

Here we must write $i \ddot{\varphi} \pi$ in terms of $\Phi$ and $\Pi$. Then, if possible, we write $Z[0]$ in Lagrangian form, either in $\Phi$ or in $\Pi$ : $Z[0]=\int[D \Phi] \exp \left\{-\int \mathrm{d} t \ell(\Phi)\right\}$

or

$$
Z[0]=\int[D \Pi] \exp \left\{-\int \mathrm{d} t \ell(\Pi)\right\} .
$$

Finally we add $-j \Phi$ or $-j \Pi$ to $\ell$ and try to work backwards.

In this paper we have only used the first option in Eq. (39), thinking of quantum fields rather than their conjugate momenta as the relevant objects. However, we could have used the second option in Section 2.2, in which case we would have finished with a simple formula like Eq. (20) for $Z[j]$, but with the roles of $\varphi$ and $\pi$ reversed. For the case of the wrong-sign quartic oscillator treated in [7], an equivalent conventional Hermitian theory, with a standard kinetic term, is only possible if $Z[0]$ is expressed in terms of $\Pi$.

In any case we have shown that $Q$ does not appear explicitly in the functional integral formalism, on the lines of Eq. (10), as might have been naively supposed. Instead the choice of metric in the operator formalism is reflected in the $j$ dependence of $Z[j]$.

It is interesting to note that in their work on the $V=\frac{1}{2} x^{2}+i g x^{3}$ model [10], Bender et al. implicitly made the assumption that $Q$ does not appear in the functional integral formalism, since their Feynman rules, for both the original theory and its Hermitian equivalent, were effectively derived from standard functional integrals. In that case $Q$ is only known perturbatively, and the series for $\ell$ involves complicated derivative couplings.

The successful construction of the equivalent Hermitian theory to that with a $-g z^{4}$ potential raises hopes that a similar construction, within the functional integral framework, might be possible for the corresponding $-g \varphi^{4}$ field theory. Some tentative steps were made in this direction in [11], but the generalization seems far from straightforward.

\section{Remarks}

(1) In this context, where $\eta$ is a positive-definite operator, the first term may be preferable. The $\mathcal{P} \mathcal{T}$ invariance of the original class of Hamiltonians (1) can be expressed as pseudo-Hermiticity with respect to the indefinite operator $P$.

\section{References}

[1] Bender, C., Boettcher, S.: Real Spectra in Non-Hermitian Hamiltonians Having PT Symmetry. Phys. Rev. Lett., Vol. 80 (1998), p. 5243-5246.

[2] Dorey, P., Dunning, C., Tateo, R.: Spectral Equivalences, Bethe Ansatz Equations, and Reality Properties in $\mathcal{P}$-symmetric Quantum Mechanics. J. Phys. Vol. A34 (2001), p. 5679-5704.

[3] Mostafazadeh, A.: Pseudo-Hermiticity versus PT Symmetry: The Necessary Condition for the Reality of the Spectrum of a Non-Hermitian Hamiltonian. J. Math. Phys. Vol. 43 (2002), p. 205-214; Exact PT -symmetry is Equivalent to Hermiticity. J. Phys. Vol. A36 (2003), p. 7081-7091.

[4] Bender, C., Brody, D., Jones, H.: Complex Extension of Quantum Mechanics. Phys. Rev. Lett. Vol. 89 (2002), 270401(1-4); ibid. Vol. 92 (2004), 119902.

[5] Scholtz, F., Geyer, H., Hahne, F.: Quasi-Hermitian Operators in Quantum Mechanics and the Variational Principle. Ann. Phys. Vol. 213 (1992), p. 74-101.

[6] Musumbu, D., Geyer, H., Heiss, W.: Choice of a Metric for the Non-Hermitian Oscillator. arXiv: quant-ph/061 1150, to be published in J. Phys. A.

[7] Jones, H., Rivers, R.: Disappearing Operator. Phys. Rev. Vol. D75 (2007), 025023 (p. 1-7).

[8] Swanson, M.: Transition Elements for a Non-Hermitian Quadratic Hamiltonian. J. Math. Phys. Vol. 45 (2004), p. 585-601.

[9] Geyer, H., Scholtz, F., Snyman, I.: Quasi-Hermiticity and the Role of a Metric in Some Boson Hamiltonians. Czech. J. Phys. Vol. 54 (2004), p. 1069-1073.

[10] Bender, C., Chen, J., Milton, K.: $\mathcal{P} \mathcal{T}$-symmetric versus Hermitian Formulations of Quantum Mechanics.J. Phys. Vol. A39 (2006), p. 1657-1668.

[11] Bender, C., Brody, D., Chen, J., Jones, H., Milton, K., Ogilvie, M.: Equivalence of a Complex $\mathcal{P T}$-symmetric Quartic Hamiltonian and a Hermitian Quartic Hamiltonian with an Anomaly. Phys. Rev. Vol. D74 (2006), 025016 (p. 1-10).

\section{Dr. Hugh F. Jones}

phone: +44 (0)2075947830

email: h.f.jones@imperial.ac.uk

Physics Department

Faculty of Natural Sciences

Imperial College London

South Kensington campus

London SW7 2AZ, United Kingdom 